\section{Custo da fluoretação das águas de abastecimento público, estudo de caso - Município de São Paulo, Brasil, período de 1985-2003}

\author{
Cost of fluoridating the public water supply: \\ a study case in the city of São Paulo, \\ Brazil, 1985-2003
}

\author{
Antonio Carlos Frias 1 \\ Paulo Capel Narvai 2 \\ Maria Ercilia de Araújo 1 \\ Celso Zilbovicius 1 \\ José Leopoldo Ferreira Antunes 1
}

\footnotetext{
1 Faculdade de Odontologia, Universidade de São Paulo São Paulo, Brasil.

2 Faculdade de Saúde Pública, Universidade de São Paulo, São Paulo, Brasil.

Correspondência A. C. Frias

Faculdade de Odontologia Universidade de São Paulo. Av. Prof. Lineu Prestes 2227 São Paulo, $S P$ 05508-900, Brasil acfrias@usp.br
}

\begin{abstract}
The aim of this study was to estimate the cost of fluoridating the public water supply in the city of São Paulo, Brazil, from 1985 to 2003. Cost calculation for fluoridation of the public water supply used the following: capital cost of initial installation, chemical product (hydrofluosilicic acid), system's operational cost (maintenance, electricity, and human resources), and monitoring fluoride levels. Fluoridation was effective, since there was a decrease of $73 \%$ in dental caries in 12-year-olds - mean DMF was 6.47 (6.12-6.82) in 1986, having decreased to 1.75 (1.48-2.92) in 2002. In this age group, $40 \%$ of children presented DMF $=0$ in 2002. Average cost per inhabitant/year for 2003 was $R \$ 0.08$ (US\$ 0.03). The accumulated cost for 18 years of implementation and maintenance of the fluoridation system was R\$1.44 (US\$ 0.97) per capita.
\end{abstract}

Fluoridation; Dental Caries; Water Supply

\section{Introdução}

A fluoretação das águas de abastecimento público é uma das medidas mais amplas de promoção de saúde na área de saúde bucal, pois associa ao benefício da água tratada o seu impacto epidemiológico na redução da prevalência e severidade da cárie dentária. A redução da severidade, medida pelo índice CPO-D, é em média de 50 a $60 \% 1,2$, porém de modo não isolado da exposição a outras fontes de flúor, como o dentifrício.

A fluoretação das águas de abastecimento público, embora seja objeto de pesquisas desde as primeiras décadas do século XX e largamente empregada em saúde pública 3 , é um tema ainda atual, uma vez que freqüentemente se apresentam questionamentos à eficácia e efetividade da medida, bem como aos custos envolvidos. Sua principal característica é reduzir o número de dentes atacados pela cárie dentária, além de não depender do componente individual para sua aplicação, pois sendo inevitável o consumo de água, o benefício ocorre de modo involuntário - basta utilizar a água para beber ou preparar os alimentos.

Cox 4 , em 1939, propôs à American Dental Association (ADA) que recomendasse oficialmente a fluoretação das águas de abastecimento público. Mas somente em 1945 ocorreu a fluoretação artificial do primeiro sistema de abastecimento de água em todo o mundo, em 
Grand Rapids, no Estado de Michigan, Estados Unidos. O primeiro município no Brasil a fluoretar suas águas de abastecimento público foi Baixo Guandu, no Espírito Santo, em 31 de outubro de 1953, sob controle do Serviço Especial de Saúde Pública (SESP). No Estado de São Paulo, Brasil, a primeira cidade a fluoretar artificialmente suas águas para consumo humano foi Marília, em 12 de dezembro de 1956. A Cidade de São Paulo teve suas águas de abastecimento público fluoretadas em 31 de outubro de 19853.

A fluoretação das águas de abastecimento, embora seja uma importante medida de saúde pública, beneficia cerca de $40 \%$ da população no Brasil 5, ou seja, $60 \%$ não têm acesso a esse benefício. No Estado de São Paulo, em 2004, 117 dos 645 municípios não fluoretavam as águas de abastecimento 6 .

Um dos fatores impeditivos, segundo alegam as companhias de abastecimento de água, seriam os custos de instalação do sistema de fluoretação e do produto químico 7,8 .

Tendo em vista essa objeção, é relevante analisar o custo da implantação e manutenção dessa importante medida de saúde pública para o controle da cárie dentária. É o que se faz neste estudo que tem como referência o Município de São Paulo, onde o sistema de fluoretação foi implantado há cerca de vinte anos, sem registro de interrupção desde então.

\section{Método}

Trata-se de um estudo de caso cujo objetivo é estimar o custo da fluoretação das águas de abastecimento público no Município de São Paulo (período de 1985-2003).

Apresenta-se os dados epidemiológicos relativos à cárie dentária disponíveis para os pe- ríodos anterior e posterior ao início da fluoretação. Todos os estudos foram transversais e realizados com base em amostras de escolares de 7 a 12 anos de idade. Estes estudos foram realizados em distintos momentos históricos visando a atender a diferentes finalidades, sendo promovidos por diversos pesquisadores e/ou órgãos governamentais, valendo-se de diferentes delineamentos amostrais e metodologias para obtenção dos dados.

Na Tabela 1 são apresentados os dados da média do índice CPO-D nas idades de 7 a 12 anos nos estudos epidemiológicos realizados no Município de São Paulo no período de 1969 a 2002 e os respectivos intervalos de confiança para 95\% (IC95\%). A Figura 1 apresenta os dados referentes às crianças de 12 anos livres de cárie dentária $(\mathrm{CPO}-\mathrm{D}=0)$ e o limite inferior e superior do IC95\%.

Levantou-se junto à Companhia de Saneamento Básico do Estado de São Paulo (SABESP) e empresas fornecedoras de equipamentos, o custo do produto químico e dos equipamentos necessários à incorporação do flúor à água de abastecimento, considerando as Estações de Tratamento de Água (ETA), responsáveis pelo abastecimento da cidade.

Para a análise dos custos, estes foram estratificados em quatro grupos: (a) custo de capital inicial de instalação; (b) custo dos produtos químicos; (c) custo de operacionalização do sistema; e (d) custo de controle dos teores de flúor, conforme apresentado no Tabela 2.

O custo de capital inicial de instalação foi composto pelos custos de equipamentos (bombas dosadoras, tanques de armazenamento, equipamentos de controle e testes de dosagem) e pelos de instalação dos equipamentos e custo de consultoria técnica. Os Custos de Instalação dos Equipamentos representam cerca de $85 \%$

Tabela 1

Média do índice CPO-D e o intervalo de confiança (IC95\%), nas idades de 7 a 12 anos dos estudos

epidemiológicos realizados. Município de São Paulo, Brasil, no período de 1969-2002.

\begin{tabular}{|c|c|c|c|c|c|c|c|c|}
\hline \multirow{2}{*}{$\begin{array}{l}\text { Idade } \\
\text { (anos) }\end{array}$} & \multicolumn{8}{|c|}{ Média CPO-D (IC95\%) } \\
\hline & 1969 & 1980 & 1982 & 1986 & $1996^{*}$ & $1996^{\star \star}$ & 1998 & 2002 \\
\hline 7 & - & $2,18(2,14-2,22)$ & $2,12(2,07-2,17)$ & $2,07(1,93-2,22)$ & $0,24(0,15-0,33)$ & $0,46(0,30-0,62)$ & $0,30(0,16-0,44)$ & - \\
\hline 8 & $2,60(2,37-2,83)$ & $3,11(3,07-3,15)$ & $2,99(2,94-3,04)$ & $2,70(2,56-2,84)$ & $0,53(0,40-0,66)$ & $0,89(0,68-1,10)$ & $0,51(0,31-0,71)$ & - \\
\hline 9 & $3,43(3,18-3,68)$ & $3,86(3,81-3,91)$ & $3,59(3,53-3,65)$ & $3,59(3,42-3,75)$ & $0,75(0,59-0,91)$ & $1,12(0,92-1,32)$ & $1,05(0,77-1,33)$ & - \\
\hline 10 & $4,47(4,14-4,80)$ & $4,59(4,52-4,66)$ & $4,43(4,35-4,51)$ & $4,11(3,88-4,34)$ & $1,15(0,94-1,36)$ & $1,34(1,10-1,58)$ & $1,12(0,85-1,39)$ & - \\
\hline 11 & $4,91(4,41-5,41)$ & $5,82(5,73-5,91)$ & $5,44(5,34-5,55)$ & $5,17(4,85-5,49)$ & $1,56(1,28-1,84)$ & $1,85(1,55-2,15)$ & $1,61(1,27-1,95)$ & - \\
\hline 12 & $6,91(6,12-7,70)$ & $7,03(6,92-7,14)$ & $6,44(6,31-6,57)$ & $6,47(6,12-6,82)$ & $2,06(1,85-2,27)$ & $2,28(1,88-2,68)$ & $2,06(1,58-2,54)$ & $1,75(1,48-2,02)$ \\
\hline
\end{tabular}

Fonte: $196926 ; 1980$ 27; 1982 27; 1986 28; 1996* 29; 1996** 30; 1998 31; 200232. 
dos custos do equipamento, segundo o Centers for Disease Control and Prevention dos Estados Unidos 9,10. Os de consultoria técnica representam 15\% dos custos de capital inicial de instalação 9,10. A composição final dos custos de capital inicial de instalação foi estratificada por vinte anos, tempo correspondente à vida útil dos equipamentos 11,12,13,14,15.

Para o custo do produto químico utilizado nas ETA que abastecem a população da Cidade de São Paulo, foram consideradas as quantidades de flúor natural que chega nos rios e mananciais e de flúor incorporado para obtenção da concentração ideal de flúor $(0,7 \mathrm{ppm})$.

Os componentes que perfazem o custo de operacionalização do sistema foram os custos de: (1) depreciação e manutenção dos equipamentos; (2) energia elétrica; e (3) recursos humanos.

O custo de depreciação e manutenção dos equipamentos representa cerca de $10 \%$ do valor total do custo de capital inicial de instalação e é distribuído pelo tempo de vida útil dos equipamentos 16 .

White et al. 16 referiram-se ao custo de energia elétrica e afirmaram que, apesar de geralmente ser difícil o cálculo e inclusão, não é apropriado excluir tais custos e estes podem ter seus valores estimados com base nos custos da ETA.

Para dimensionar o custo de energia elétrica com a fluoretação foi utilizada a porcentagem dos gastos de energia elétrica de toda a rede de abastecimento, sendo aplicada a mesma porcentagem sobre os custos do produto químico utilizado para a fluoretação.

Para estimar os custos com recursos humanos, levou-se em consideração os custos com funcionários do nível operacional do sistema de fluoretação e os custos com outros funcionários para o controle e testes laboratoriais. Alem do salário, foram considerados os encargos trabalhistas com férias, 13o salário, previdência e seguro-trabalho.

O controle operacional do sistema de fluoretação é realizado com os mesmos recursos humanos de operação do sistema de tratamento de água não onerando, assim, o custo final. O controle laboratorial é exercido por pessoal técnico especializado que realiza, além do controle do flúor, outros testes químicos para controle da qualidade da água. Assim, considerouse um funcionário para cada ETA para dimensionar o custo com recursos humanos.

O custo de controle dos teores de flúor na água de abastecimento corresponde a um valor fixo representado pelo equipamento de medição e controle, cujo valor foi acrescido ao custo de capital inicial de instalação. A esse va-

\section{Figura 1}

Porcentagem de crianças com CPO-D = 0, IC95\% para a média populacional, na idade de 12 anos no período de 1969 a 2002. São Paulo, Brasil.

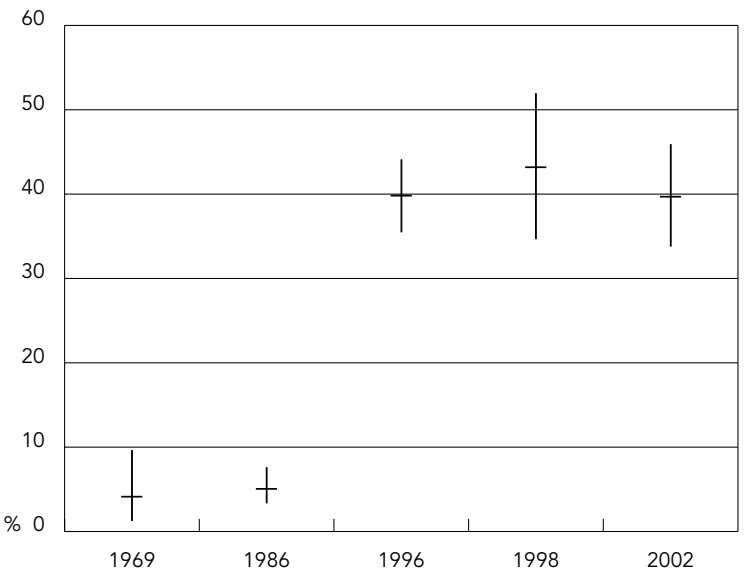

Fonte: 1969 26; 1986 28; 1996 29; 1998 32; 200233.

lor fixo agregou-se um custo continuo, representado pelos eletrodos de íon-seletivo para a dosagem de fluoreto que têm, em média, um ano de vida útil 9 .

O "indicador de custo da fluoretação", que expressa o valor do custo de fluoretação per capita/ano, foi obtido mediante a seguinte fórmula:

Indicador de custo

da fluoretação $=\frac{\mathrm{CCII}+\mathrm{CPQ}+\mathrm{COS}+\mathrm{CCF}}{\text { População da cidade no ano }}$

onde: $\mathrm{CCII}=$ custo de capital inicial de instalação; $\mathrm{CPQ}=$ custo de produto químico; $\mathrm{COS}=$ custo de operacionalização do sistema; $\mathrm{CCF}=$ custo de controle dos teores de flúor.

Para a realização dos cálculos de custos empreendidos neste trabalho, utilizaram-se os dados referentes aos custos de tratamento de água da ETA Guaraú, (custos de produtos químicos, ácido fluorsilícico, custos com recursos humanos, com energia elétrica etc.), obtidos por meio de relatórios internos de custos mensais no período de 1998 a 2003. Como o volume de água tratada $\left(33 \mathrm{~m}^{3} / \mathrm{seg}\right)$ e consumo de ácido fluorsilícico (10 toneladas por dia) na ETA Guaraú representam aproximadamente 50\% do consumo do município $\left(64 \mathrm{~m}^{3} / \mathrm{seg}\right.$ de água tratada e 19,3 toneladas de ácido fluorsilícico), os valores encontrados na ETA Guaraú foram dobrados. Este recurso metodológico foi empregado visando a projetar o consumo total no 
Composição dos custos de fluoretação das águas de abastecimento.

\begin{tabular}{|c|c|c|}
\hline \multicolumn{3}{|c|}{ Composição de custos } \\
\hline $\mathrm{CCl}$ & Custo de equipamentos & $\begin{array}{l}\text { Bombas, tanques de armazenamento, } \\
\text { Equipamento de controle (fluorímetro) }\end{array}$ \\
\hline & Custo de instalação & $85 \%$ custos dos equipamentos \\
\hline & Custo de consultoria técnica & $15 \% \mathrm{CCII}$ \\
\hline \multicolumn{3}{|c|}{ Fracionados por vinte anos - tempo de vida útil do equipamento } \\
\hline $\mathrm{CPO}$ & $\begin{array}{l}\text { Flúor natural + flúor incorporado no sistema. } \\
\text { Teor de flúor ideal }(0,7 \text { ppm). }\end{array}$ & $\begin{array}{l}\text { Custo da tonelada de ácido fluorsilícico } \\
\text { utilizado nas ETA }\end{array}$ \\
\hline \multirow[t]{3}{*}{$\cos$} & Custo de depreciação e manutenção & $10 \%$ CCII - fracionados por vinte anos \\
\hline & Custo de energia elétrica & 5 a $8 \%$ do custo do AFS \\
\hline & Recursos humanos & $\begin{array}{l}\text { Salário e encargos de um técnico } \\
\text { por estação de tratamento de água }\end{array}$ \\
\hline $\mathrm{CCF}$ & Eletrodos de flúor & 1 para cada ETA do sistema \\
\hline
\end{tabular}

$\mathrm{CCII}=$ custo de capital inicial de instalação; $\mathrm{CPQ}=$ custo dos produtos químicos;

$\mathrm{COS}$ = custo de operacionalização do sistema; CCF = custo de controle dos teores de flúor;

$E T A=$ estações de tratamento de água.

município, uma vez que nem todas as ETA possuem relatórios detalhados de seus custos operacionais do sistema de tratamento.

Para o cálculo dos custos de fluoretação foram obtidas informações dos fornecedores de equipamentos e utilizados dados dos relatórios da SABESP (Companhia de Saneamento Básico do Estado de São Paulo), aos quais aplicou-se a fórmula do indicador de custo da fluoretação. Assim, foram estimados os custos do siste$\mathrm{ma} / \mathrm{ano}$ e por pessoa/ano.

Os custos foram atualizados para o ano-base de 2003 tendo como índice de correção monetária o Índice de Preços ao Consumidor (IPC) da Fundação Instituto de Pesquisa Econômica da Universidade de São Paulo (FIPE/ USP - http:/ / www.ocaixa.com.br/bancodedados/ipe-fipe.htm, acessado em 20/Fev/2004).

Para estimar os custos acumulados do sistema de fluoretação no período anterior (19851997), calculou-se o custo médio de ácido fluorsilícico em 2003 ajustado monetariamente pelo IPC/FIPE. Calculou-se a porcentagem de participação dos custos do ácido fluorsilícico sobre os custos totais da fluoretação tendo como referência os custos no período de 1998-2003.

Utilizando-se os relatórios da SABESP, obteve-se o volume de ácido fluorsilícico consumido mensalmente no período de 1985-1997. Foi possível, então, estimar o custo da fluoretação, pois o volume de ácido fluorsilícico consumido no período foi multiplicado pelo valor do custo médio do ácido fluorsilícico de 2003, obtendo-se o custo atualizado do ácido fluorsilícico. Considerando-se que esse ácido representa uma porcentagem dos custos da fluoretação, estimou-se o custo anual do sistema no período de 1985-1997.

O Brasil passou por períodos de grandes instabilidades financeiras entre 1985 e 1993, trocando sua moeda corrente cinco vezes devido à inflação. Em agosto de 1994 foi criado o Real que é utilizado até os dias atuais. Desde então, a economia esteve relativamente estabilizada. A moeda brasileira teve, no período de 1994-2003, em relação ao dólar, uma taxa de conversão estável, onde a variação média, real/dólar, neste período de nove anos foi de $\mathrm{R} \$ 1,72$ por US\$1,00. O dólar americano (US\$) foi usado como moeda de referência, pois, segundo Niessen \& Douglass 17, o dólar é uma ótima referência monetária, podendo ser utilizado na apresentação de cálculo de custos do tipo realizado neste estudo.

Para estimar os custos de 1985-1997, este foi dividido em dois períodos, antes da implantação do real (1985-1993) e pós-implantação do real (1994-1997). Para o período de 19941997 projetou-se os custos em reais para os custos de consumo ácido fluorsilícico e para os de implantação do sistema de fluoretação. Posteriormente este valor foi convertido em dólar americano pela média anual praticada no mercado financeiro.

Em relação à estimativa de custos no período de 1985-1993, este foi projetado em real, de- 
vido à grande variação monetária no período, para os custos de consumo de ácido fluorsilícico e para os de implantação do sistema de fluoretação. Em seguida os valores foram convertidos para o dólar, tendo como taxa de conversão real/dólar a cotação da variação média no período de 1994-2003, ou seja, R\$ 1,72/US\$1,00.

Adotou-se a projeção do crescimento da população da Cidade de São Paulo, segundo uma taxa de 0,91 no período de 1991-2000, conforme preconizado pela Fundação SEADE 18. A mesma taxa foi utilizada para os anos de 2001-2003. Utilizou-se também o "Método de crescimento médio anual" usando-se a taxa de progressão de crescimento aritmético proposto por Laurenti et al. 19. Calculou-se o custo per capita/ano em relação ao real e ao dólar (cotação média do dólar no ano em relação ao real), obtendo-se o custo da fluoretação per capita/ano em dólar, conforme o "indicador de custos da fluoretação".

É relevante assinalar que, em termos metodológicos, focaliza-se o estudo na estimativa do custo da fluoretação operando-se a análise num contexto típico de "experimento social", onde não é possível isolar e controlar todas as variáveis intervenientes num determinado processo que produz um certo desfecho, neste caso o perfil da cárie dentária.

\section{Resultados}

A Região Metropolitana de São Paulo conta com oito sistemas interligados de abastecimento de água, sendo que cinco destes são destinados ao abastecimento da Cidade de São Paulo: ETA Guaraú, Alto da Boa Vista, Alto Tietê, Rio Grande e Rio Claro.

O consumo médio diário de ácido fluorsilícico $\left(\mathrm{H}_{2} \mathrm{SiF}_{6}\right)$ pela população da Cidade de São Paulo é de 19,3 toneladas. Segundo os relatórios de controle dos cinco sistemas, a concentração de flúor natural vinda dos rios e mananciais que abastecem as ETA é de aproximadamente $0,1 \mathrm{ppm}$ de flúor, tornando-se necessária a adição de 0,6 ppm para se obter o teor final (teor ótimo) de 0,7ppm.

O custo de capital inicial de instalação correspondeu aos custos: (a) dos equipamentos: dez bombas dosadoras ( $\mathrm{R} \$ 40.000,00)$ e 31 tanques de armazenamento do ácido fluorsilícico ( $R \$ 49.600,00$ ); dos equipamentos de controle dos níveis de flúor realizados nos laboratórios das ETA: o medidor de bancada de eletrodos com íon-seletivo de flúor ("Fluorímetro") teve custo de $\mathrm{R} \$ 6.345,00$ (para as cinco ETA o custo total foi de R\$31.725,00), o me- didor de flúor do tipo on-line cujo custo foi de $\mathrm{R} \$ 24.700,00$ (apenas na ETA Guaraú). O custo total dos equipamentos de testes e controle foi de R \$ 56.425,00; (b) de instalação dos equipamentos, que representaram cerca de $85 \%$ do valor dos equipamentos (R\$ 124.121,25); (c) de consultoria técnica, que representaram aproximadamente $15 \%$ dos custos de capital inicial de instalação ( $\mathrm{R} \$ 40.521,94)$. Assim, o custo total no item capital inicial de instalação foi de R $\$ 270.146,25$. Fracionando-o por vinte anos, tais custos correspondem a $\mathrm{R} \$ 15.533,41$ por ano.

O custo do produto químico refere-se ao custo do capital inicial de instalação. O custo por tonelada entre 1998 e 2003 sofreu uma variação média de preço anual por tonelada de $\mathrm{R} \$ 97,50$ a $\mathrm{R} \$ 60,83$ sendo que, em dezembro de 2003, a tonelada de ácido fluorsilícico era comercializada a $\mathrm{R} \$ 100,00$, ou seja, $\mathrm{R} \$ 0,01$ por $\mathrm{kg}$ de ácido fluorsilícico. Este valor inclui o custo de transporte do produto da empresa produtora até a respectiva ETA.

Neste estudo optou-se por utilizar como base a média de custo de ácido fluorsilícico no ano de 2003 (R\$ 91,40 por tonelada), para o cálculo do custo do ácido fluorsilícico no período de 1985-1997.

No âmbito do custo de operacionalização do sistema, o de depreciação e de manutenção dos equipamentos representa $10 \%$ dos custos de capital inicial de instalação, ou seja, R\$1.553,34 ao ano em nosso caso. Para o cálculo dos custos com energia elétrica para a fluoretação, utilizando-se a porcentagem dos gastos com energia elétrica de toda a rede de abastecimento e aplicando-se este porcentual ao custo do ácido fluorsilícico utilizado na ETA obtém-se: em 1998, 5,16\% = R\$ 35.181,90; em $1999,5,54 \%=\mathrm{R} \$ 35.310,56$; em 2000, 5,73\% = $\mathrm{R} \$ 35.162,05$; em 2001, 5,87\% = R\$ 30.627,86; em 2002, $7,13 \%=\mathrm{R} \$ 38.295,92$; e em 2003, $8,23 \%=\mathrm{R} \$ 54.943,85$.

Ainda com relação aos custos com a operacionalização do sistema, o custo com recursos humanos foi obtido tomando-se como base que um funcionário técnico que trabalha na função de controle laboratorial recebe um salário de $\mathrm{R} \$$ $1.500,00$ mensais mais 13o salário, $\mathrm{R} \$ 675,00 \mathrm{de}$ encargos trabalhistas e R\$450,00 de 1/3 de férias, totalizando $\mathrm{R} \$ 28.725,00$ /ano por ponto de fluoretação. Anualmente o custo com recursos humanos, para as cinco ETA, na Cidade de São Paulo, foi estimado em R \$ 143.625,00.

O custo de controle de flúor correspondeu ao do eletrodo de íon-seletivo para a dosagem de fluoreto, equivalente a R\$1.500,00. Admitindo-se a necessidade técnica da troca anual 
desse eletrodo, por estação de tratamento, chega-se a um custo total de $\mathrm{R} \$ 7.500,00$ por ano para o sistema de controle (Tabela 3 ).

O custo do AFS no período de 1998 a 2003 representou, em média, 74,59\% dos custos totais da fluoretação. Multiplicando-se este valor pelo custo do ácido fluorsilícico nos anos de 1985 a 1997 obtém-se os custos anuais de fluoretação (Tabela 4).

\section{Discussão}

A importância da análise de custos da fluoretação no âmbito das políticas públicas de saúde é inegável, pois podem subsidiar tecnicamente municípios que queiram implantar a fluoretação, como também dar sustentação à manu- tenção da medida nos municípios cujas populações contam com o benefício. Sendo a fluoretação das águas uma medida de saúde pública de acesso potencialmente universal, é indispensável analisar seu custo.

Pode-se observar que no período 19691986 a cárie dentária mantinha-se em um patamar elevado, sem ocorrerem mudanças estatisticamente significativas; em 1986 o valor do índice CPO-D era de 6,74 $(6,12-6,82)$ em crianças de 12 anos de idade, com incremento médio era de 0,88 dentes atacados por cárie a cada ano de vida, e apenas $5 \%$ das crianças nessa idade não tinham experiência de cárie dentária na dentição permanente (CPO-D igual a zero).

Após 16 anos (2002), constatou-se uma redução da ordem de $73 \%$ no valor do índice

Tabela 3

Composição dos custos de fluoretação das águas de abastecimento público no Município de São Paulo, Brasil,

no período de 1998-2003.

\begin{tabular}{|c|c|c|c|c|c|c|}
\hline & \multicolumn{6}{|c|}{ Anos } \\
\hline & 1998 & 1999 & 2000 & 2001 & 2002 & 2003 \\
\hline $\begin{array}{l}\text { Custo de capital inicial } \\
\text { de instalação (R\$) }\end{array}$ & $15.533,41$ & $15.533,41$ & $15.533,41$ & $15.533,41$ & $15.533,41$ & $15.533,41$ \\
\hline Percentual dos custos (\%) & 1,75 & 1,85 & 1,90 & 2,16 & 2,09 & 1,74 \\
\hline Custo químico (R\$) & $681.819,86$ & $637.374,69$ & $613.648,28$ & $521.769,29$ & $537.109,64$ & $667.604,54$ \\
\hline Percentual dos custos (\%) & 77,02 & 75,80 & 75,11 & 72,41 & 72,23 & 74,95 \\
\hline $\begin{array}{l}\text { Custos de operacionalização } \\
\text { do sistema ( } R \$ \text { ) }\end{array}$ & $180.360,25$ & $180.488,90$ & $180.340,39$ & $175.806,20$ & $183.474,26$ & $200.112,19$ \\
\hline Percentual dos custos (\%) & 20,37 & 21,46 & 22,07 & 24,40 & 24,67 & 22,47 \\
\hline Custo de controle (R\$) & $7.500,00$ & $7.500,00$ & $7.500,00$ & $7.500,00$ & $7.500,00$ & $7.500,00$ \\
\hline Percentual dos custos (\%) & 0,85 & 0,89 & 0,92 & 1,04 & 1,01 & 0,84 \\
\hline Custo total (R\$) & $882.179,96$ & $837.863,45$ & 813.988 .43 & $717.575,35$ & $740.583,76$ & $887.726,59$ \\
\hline Volume de água tratada & 2.075 .595 .956 & 2.091 .136 .994 & 2.039 .043 .280 & 1.916 .249 .234 & 2.019 .993 .450 & 1.992 .771 .688 \\
\hline População & 10.231 .378 & 10.314 .977 & 10.398 .576 & 10.482 .175 & 10.565 .774 & 10.649 .373 \\
\hline $\begin{array}{l}\text { Porcentagem de atualização } \\
\text { monetária IPC/FIPE (\%) }\end{array}$ & $-1,79$ & 8,64 & 4,38 & 7,13 & 9,92 & 8,17 \\
\hline $\begin{array}{l}\text { Cotação média ano } \\
\text { US\$/R\$ }\end{array}$ & $\begin{array}{r}\text { US\$1,00/ } \\
\mathrm{R} \$ 1,164\end{array}$ & $\begin{array}{r}\text { US\$ } 1,00 / \\
\mathrm{R} \$ 1,851\end{array}$ & $\begin{array}{r}\text { US\$ } 1,00 / \\
\mathrm{R} \$ 1,835\end{array}$ & $\begin{array}{r}\text { US\$ } 1,00 / \\
\mathrm{R} \$ 2,353\end{array}$ & $\begin{array}{r}\text { US\$ } 1,00 / \\
\mathrm{R} \$ 2,998\end{array}$ & $\begin{array}{r}\text { US } \$ 1,00 / \\
\mathrm{R} \$ 3,063\end{array}$ \\
\hline $\begin{array}{l}\text { Custo/população } \\
\text { pessoa/ano (R\$) }\end{array}$ & 0,087 & 0,082 & 0,079 & 0,069 & 0,070 & 0,084 \\
\hline $\begin{array}{l}\text { Custo/população } \\
\text { pessoa/ano (US\$) }\end{array}$ & 0,074 & 0,044 & 0,043 & 0,029 & 0,024 & 0,028 \\
\hline
\end{tabular}

IPC = índice de preços ao consumidor; FIPE = Fundação Instituto de Pesquisa Econômica. 
Custos de fluoretação das águas de abastecimento público no período de 1985-2003 no Município de São Paulo, Brasil.

\begin{tabular}{|c|c|c|c|c|c|c|c|}
\hline Ano & $\begin{array}{l}\text { Ácido fluorsilícico } \\
\text { (toneladas) }\end{array}$ & $\begin{array}{c}\text { Custo ácido } \\
\text { fluorsilícico (R\$) }\end{array}$ & Custo total (R\$) & População & $\begin{array}{c}\text { Custo pessoa/ } \\
\text { ano (R\$) }\end{array}$ & Relação US\$/R\$* & $\begin{array}{c}\text { Custo pessoa/ } \\
\text { ano (US\$) }\end{array}$ \\
\hline $1985^{\star \star}$ & 720 & $65.808,00$ & $88.226,30$ & 8.265 .134 & 0,011 & 1,715 & 0,006 \\
\hline 1986 & 4.646 & $424.644,40$ & $569.304,73$ & 8.495 .309 & 0,067 & 1,715 & 0,039 \\
\hline 1987 & 5.474 & $500.323,60$ & $670.764,98$ & 8.725 .484 & 0,077 & 1,715 & 0,045 \\
\hline 1988 & 5.530 & $505.442,00$ & $677.627,03$ & 8.955 .659 & 0,076 & 1,715 & 0,044 \\
\hline 1989 & 5.562 & $508.366,80$ & $681.548,20$ & 9.185 .835 & 0,074 & 1,715 & 0,043 \\
\hline 1990 & 5.730 & $523.722,00$ & $702.134,33$ & 9.416 .010 & 0,075 & 1,715 & 0,043 \\
\hline 1991 & 6.168 & $563.755,20$ & $755.805,34$ & 9.646 .185 & 0,078 & 1,715 & 0,046 \\
\hline 1992 & 5.816 & $531.582,40$ & $712.672,48$ & 9.729 .784 & 0,073 & 1,715 & 0,043 \\
\hline 1993 & 6.690 & $611.466,00$ & $819.769,41$ & 9.813 .383 & 0,084 & 1,715 & 0,049 \\
\hline 1994 & 6.988 & $638.703,20$ & $856.285,29$ & 9.896 .983 & 0,087 & 0,870 & 0,099 \\
\hline 1995 & 7.490 & $684.586,00$ & $917.798,63$ & 9.980581 & 0,092 & 0,923 & 0,100 \\
\hline 1996 & 7.486 & $684.220,40$ & $917.308,49$ & 10.0641 .80 & 0,091 & 1,008 & 0,090 \\
\hline 1997 & 7.370 & $673.618,00$ & $903.094,25$ & 10.147.779 & 0,089 & 1,081 & 0,082 \\
\hline 1998 & 7.004 & $681.819,86$ & $885.213,52$ & 10.231 .378 & 0,087 & 1,164 & 0,074 \\
\hline 1999 & 7.468 & $637.374,69$ & $840.897,00$ & 10.314 .977 & 0,082 & 1,851 & 0,044 \\
\hline 2000 & 7.400 & $613.648,28$ & $817.022,08$ & 10.398 .576 & 0,079 & 1,835 & 0,043 \\
\hline 2001 & 7.316 & $521.769,29$ & $720.608,90$ & 10.482 .175 & 0,069 & 2,353 & 0,029 \\
\hline 2002 & 7.340 & $537.109,64$ & $743.617,31$ & 10.565 .774 & 0,070 & 2,998 & 0,024 \\
\hline 2003 & 7.328 & $667.604,54$ & $890.760,14$ & 10.649 .373 & 0,084 & 3,063 & 0,028 \\
\hline Total & 119.526 & $10.575 .564,30$ & $14.170 .458,41$ & - & 1,442 & - & 0,971 \\
\hline Média & 6.291 & $556.608,65$ & $745.813,60$ & - & 0,076 & - & 0,051 \\
\hline
\end{tabular}

* Fonte: Ribeiro PC. Cotação do Dólar comercial venda. Gazeta Mercantil/Valor Econômico.

http://www.geocities.com/Paris/rue/5045/DOLARM.HTM (acessado em 24/Jan/2004).

$\star \star 1985=$ meses de novembro e dezembro.

CPO-D, registrando-se 1,75 (1,48-2,02); aos 12 anos de idade, nos levantamentos epidemiológicos realizados no período de 1996-2002, observa-se que a porcentagem de crianças livres de cárie dentária elevou-se para cerca de $40 \%$, nessa idade.

Dentre as variáveis que podem ter influenciado os padrões da cárie dentária nestes últimos 16 anos, podemos destacar: a exposição da população à fluoretação das águas de abastecimento desde 1985, pela exposição a dentifrícios fluoretados, com mais intensidade a partir de 1988 onde quase $100 \%$ dos dentifrícios estão fluoretados 20 , bem como as ações coletivas de saúde com caráter preventivo/educativo da população, impulsionadas na cidade com a implantação do SUS e a municipalização da saúde 3 .

Com a análise dos custos, observou-se que o gasto para proteger um morador da Cidade de São Paulo no período de 1985-2003, desde a implantação do sistema de fluoretação foi de $\mathrm{R} \$ 14.170 .458,41$, o que equivale a R 1,44 (US\$ $0,97)$ por habitante. Este valor é expressivamente menor que o custo de uma restauração dentária (uma face, de amálgama, por exemplo) ou de uma extração dentária, confirmando-se, assim, o baixo custo da medida frente ao importante benefício que ela proporciona.

Os custos com equipamentos e sua respectiva instalação (custos de capital inicial de instalação) foram de R $\$ 15.533,41$ (US\$ 5.071,31) por ano para as cinco ETA ou US\$ $1.014,26$ por ETA. Para Niessen \& Douglass 17, o custo encontrado foi de US\$ 6.000,00 por ponto de tratamento e, portanto, o valor encontrado na Cidade de São Paulo foi relativamente bem menor. Por certo as diferenças entre as economias dos diferentes países implicam cautela na interpretação desses resultados. Manau et al. 12 calcularam o custo dos equipamentos, desde a instalação, para um ponto de fluoretação e encontraram o valor de US\$ 58.333,00 (em vinte anos). Custos desse tipo para o presente estudo de caso em São Paulo foram duas vezes menores, correspondendo a R\$ $54.029,25$ por ETA (ou US\$ $31.503,94$ ) para um período de 18 anos. Horowitz \& Heifetz 21, obtiveram um custo de US\$ 0,05 por pessoa/ 
ano para os equipamentos. Em São Paulo esses custos foram de R \$ 0,001 (US\$ 0,0005).

Garcia 13 estimou o custo da fluoretação para comunidades com mais de 200 mil habitantes nos Estados Unidos e obteve um custo de US\$ 0,12 per capita/ano. Ringelberg et al. 14 calcularam o custo da fluoretação para 44 comunidades da Flórida (Estados Unidos) obtendo US $\$ 0,45$ por pessoa/ano. Newbrun 22 calculou o custo da fluoretação na década de 80 nos Estados Unidos, encontrando valores que oscilaram entre US $\$ 0,25$ e 0,50 por pessoa/ ano. O custo médio obtido para a Cidade de São Paulo, entre 1985 e 2003, foi de US\$ 0,03 per capita/ano, um valor cerca de dez vezes menor.

No Brasil, Barbisan et al. 23 estimaram o custo da fluoretação na Cidade de Curitiba, no ano de 1968, em Cr\$ 0,29 por família/ano e em Cr\$ 0,06 por pessoa/ano. Para a composição dos custos, os autores incluíram gastos com produtos químicos (flúor) e energia elétrica e não computaram gastos com recursos humanos. Luce 24 projetou o custo para o Brasil em Cr\$ 1,00 (ou US\$ 0,06) por pessoa/ano e, para o Estado do Rio Grande do Sul, em Cr\$ 1,50 (ou US\$ 0,09) por pessoa/ano. Segundo dados da Secretaria de Estado da Saúde do Paraná, o custo da fluoretação das águas nas cidades paranaenses foi de $\mathrm{R} \$ 1.089 .602,14$ em 2000, para atender 7.269.947 habitantes, o que representou um custo de $\mathrm{R} \$ 0,15$ por pessoa/ano e de $\mathrm{R} \$ 0,75$ por família/ano 25 .

Comparando-se essas estimativas de custos com os resultados encontrados para o Município de São Paulo, observa-se que os valores obtidos por Luce 24 para o Brasil (US\$ 0,06) são duas vezes maiores e os estimados para o Rio Grande do Sul (US\$ 0,09) são três vezes maiores. Em relação aos custos nas cidades do Paraná, os valores são duas vezes maiores que os encontrados para a Cidade de São Paulo.

Segundo Calvo 7 e Pinto ${ }^{8}$, os custos relativos à aquisição e instalação dos equipamentos são tidos como impeditivos para a implantação da fluoretação. Observa-se, contudo, que estes representaram, em média, 1,92\% dos custos totais no estudo da Cidade de São Paulo.

Os custos com recursos humanos $(22,6 \%$ dos custos totais) e os com o produto químico - ácido fluorsilícico (74,6\% dos custos totais) representaram $97,2 \%$ dos custos totais. É admissível, portanto, que sendo os custos com pessoal inerentes ao tratamento da água, o item de custo decisivo para a implantação e manutenção da medida seja o relacionado com o produto químico, uma vez que, isoladamente, esse item corresponde a três quartos dos custos totais. Tal peso relativo, é de admitir, poderia explicar boa parte dos casos de interrupção da fluoretação, em contextos onde a eficácia e a segurança da medida não são questionadas e, portanto, não justificam a interrupção.

Além do benefício diretamente ligado à redução nos níveis de cárie dentária, cabe assinalar a decorrente diminuição na demanda por assistência individual de urgência, acarretando menor absenteísmo escolar e no trabalho, além da economia financeira em casos de necessidade de tratamentos mais complexos.

A fluoretação das águas é uma ação de alta eficiência, baixo custo relativo e grande benefício social. Neste estudo de caso constatou-se que, a um custo per capita social e economicamente suportável, é uma das variáveis que atuam na transformação significativa do perfil epidemiológico da cárie, alem de diminuir a desigualdade social no acesso a um produto fluorado, beneficiando indistintamente todos os estratos da população. A medida contribui também para melhorar a qualidade de vida, ajudar a aumentar a auto-estima das pessoas e evitar o constrangimento de, ao sorrir, envergonhar-se de mostrar arcos dentários mutilados ou com dentes parcialmente destruídos. Tais benefícios, porém, são intangíveis não podendo ser estimados, uma vez que seu valor diz respeito a aspectos de subjetividade não alcançados por modelos avaliativos de base monetária, como o empregado neste estudo. 


\section{Resumo}

O objetivo do artigo é estimar o custo da fluoretação das águas de abastecimento público no Município de São Paulo no período de 1985-2003. Para o cálculo da composição de custos da fluoretação das águas levouse em conta os seguintes custos: (a) capital inicial de instalação; (b) produto químico (ácido fluorsilícico); (c) operacionalização do sistema (manutenção do sistema, energia elétrica e recursos humanos); $\boldsymbol{e}(d)$ controle dos teores de flúor. A ação do flúor mostrou-se efetiva, pois para a idade de 12 anos observou-se redução de $73 \%$ na experiência de cárie dentária, sendo que em 1986 a média do índice CPO-D era de 6,47 $(6,12-6,82)$ e em 2002 foi de $1,75(1,48-2,92)$. Nesta idade-índice $40 \%$ das crianças apresentaram CPO-D $=0$ em 2002. O custo médio per capita/ano na Cidade de São Paulo foi de $R \$ 0,08$ (US\$ 0,03) em 2003. O custo acumulado em 18 anos de implantação do sistema de fluoretação foi de $R \$ 1,44$ (US\$ 0,97) per capita.

Fluoração; Cárie Dentária; Abastecimento de Água

\section{Colaboradores}

A. C. Frias contribuiu com o planejamento e a elaboração do projeto de pesquisa, a coleta de dados, a revisão da literatura, a análise de custos e redação do artigo. P. C. Narvai contribuiu com o planejamento e coordenação da pesquisa, com a redação do artigo e revisão do texto final. M. E. Araújo colaborou na redação do artigo e revisão do texto final. C. Zilbovicius colaborou na revisão da literatura, na tradução dos artigos e na redação do artigo. J. L. F. Antunes colaborou na análise dos custos e na redação final do texto.

\section{Agradecimentos}

Os autores agradecem à Companhia de Saneamento Básico do Estado de São Paulo por gentilmente permitir o acesso aos relatórios técnicos da empresa, bem como ao Engenheiro Alexandre Saron pela solicitude com que, prontamente, colaborou com os pesquisadores no trabalho que deu origem a este artigo. Ao Prof. Dr. Jaime A. Cury (Universidade Estadual de Campinas), ao Dr. Getulio Martins (Associação Brasileira de Engenharia Sanitária e Ambiental - São Paulo) e ao professor Flávio Antonio Luce (Universidade Federal do Rio Grande do Sul), pela cessão de documentos, separatas e preciosas sugestões.

\section{Referências}

1. Ripa LW. A half-century of community water fluoridation in the United States: review and commentary. J Public Health Dent 1993; 53:17-62.

2. Bastos RS, Olympio KPK, Bijella VT, Buzalaf MAR, Bastos JRM. Trends in dental caries prevalence in 12-year-old schoolchildren between 1876 and 2001 in Bauru, Brasil. Public Health 2005; 199: 269-75.

3. Narvai PC. Cárie dentária e flúor: uma relação do século XX. Ciênc Saúde Coletiva 2000; 5:381-92.

4. Cox GJ. New knowledge of fluorine in relation to dental caries. J Am Water Works Assoc 1939; 31: 926.

5. Instituto Brasileiro de Geografia e Estatística. Atlas de saneamento. http://www.ibge.gov.br/ (acessado em 13/Abr/2004).

6. Secretaria de Estado da Saúde de São Paulo. Projeto promoção e qualidade de vida - fluoretação das águas de abastecimento. São Paulo: Secretaria de Estado da Saúde de São Paulo; 2004.

7. Calvo MCM. Situação da fluoretação de águas de abastecimento público no Estado de São Paulo. Brasil [Dissertação de Mestrado]. São Paulo: Faculdade de Saúde Pública, Universidade de São Paulo; 1996.

8. Pinto VG. Saúde bucal coletiva. São Paulo: Editora Santos; 2000.

9. Centers for Disease Control and Prevention. Water fluoridation: a manual for engineers and technicians. Atlanta: US Department of Health and Human Services, Public Health Service, Centers for Disease Control and Prevention; 1991.

10. Centers for Disease Control and Prevention. Engineering and administrative recommendation for water fluoridation, 1995. MMWR Recomm Rep 1995; 44(RR-13):1-40.

11. Nelson W, Swint JM. Cost-benefit analysis of fluoridation in Houston, Texas. J Public Health Dent 1976; 36:88-95.

12. Manau C, Cuenca E, Martinez-Carretero J, Salleras L. Economic evaluation of community programs for the prevention of dental caries in Catalonia, Spain. Community Dent Oral Epidemiol 1987; 15: 297-300.

13. Garcia AI. Caries incidence and cost of preventive programs. J Health Dent 1989; 49(5 Spec No):25971 .

14. Ringelberg ML, Allen SJ, Brown LJ. Cost of fluoridation: 44 Florida communities. J Public Health Dent 1992; 52:75-80.

15. Wright JC, Bates MN, Cutress T, Lee M. The costeffectiveness of fluoridating water supplies in New Zealand. Australian and New Zealand. J Public Health 2001; 25:170-8.

16. White BA, Antczak-Bouckoms AA, Weinstein MC. Issues in the economic of community water fluoritation. J Dental Educ 1989; 53:646-57.

17. Niessen L, Douglass CW. Theoretical considerations in applying benefit-cost and cost-effectiveness analyses to preventive dental programs. J Public Health Dent 1984; 44:156-68.

18. Fundação Sistema Estadual de Análise de Dados. Perfil Municipal de São Paulo http://www.seade. gov.br/perfil/index.htmt (acessado em 08/Out/ 2003). 
19. Laurenti R, Mello Jorge MHP, Lebrão ML, Gotlieb SLD. Estatísticas de saúde. 2a Ed. São Paulo: EPU; 1987.

20. Cury JA, Tabchoury CPM, Determination of appropriate exposure to fluoride in non-EME countries in the future. J Appl Oral Sci 2003; 11:83-95.

21. Horowitz HS, Heifetz SB. Methods for assessing the cost-effectiveness of caries preventive agents and procedures. Int Dental J 1979; 29:106-17.

22. Newbrun E. The fluoridation war: a scientific dispute or a religious argument? J Public Health Dent 1996; 56(5 Spec No):246-51.

23. Barbisan AO, Ribeiro CRM, Fossati GF, Caminha JAN, Edler OS. Implicação econômica - familiar decorrente da fluoretação de águas de abastecimento público no Rio Grande do Sul. Rev Fac Odontol P Alegre 1970; 12:35-44.

24. Luce FA. Fluoretação da água no Rio Grande do Sul. In: Emmerich A, Freire AS, organizadores. Flúor e saúde coletiva - 50 anos de fluoretação da água no Brasil. Vitória: Editora da UFES; 2003. p. 69-74.

25. Secretaria de Estado de Saúde do Paraná. Custo da fluoretação das águas de abastecimento Estado do Paraná em 2000. http://www.saude.pr.gov. $\mathrm{br/bucal/fluoretacao/custo-fluoretacao.htm}$ (acessado em 10/Out/ 2003).

26. Souza JMP. Índice CPO-D, índice de ataque, número de dentes irrompidos: Comportamento em escolares do Município de São Paulo [Tese de Doutorado]. São Paulo: Faculdade de Saúde Pública, Universidade de São Paulo; 1970.
27. Rosa AGF. Características epidemiológicas da cárie dental na dentição permanente de escolares do grupo etário de 7 a 14 anos no Estado de São Paulo [Tese de Doutorado]. São Paulo: Faculdade de Saúde Pública, Universidade de São Paulo; 1987.

28. Ministério da Saúde. Levantamento epidemiológico em saúde bucal: Brasil, zona urbana, 1986. Brasília: Divisão Nacional de Saúde Bucal, Secretaria Nacional de Programas Especiais de Saúde, Ministério da Saúde; 1988.

29. Secretaria de Estado da Saúde de São Paulo, Universidade de São Paulo. Levantamento epidemiológico da cárie dentária, oclusopatias e fluorose dentária, em crianças de 5 a 12 anos de idade, em escolas públicas e privadas do Município de São Paulo, em 1996. São Paulo: Faculdade de Saúde Pública, Universidade de São Paulo; 1997.

30. Ministério da Saúde. Levantamento epidemiológico em saúde bucal - 1996: primeira etapa cárie dental. http://www.datasus.gov.br/cgi/sbu$\mathrm{cal} /$ sbdescr.htm (acessado em 17/Out/2002).

31. Secretaria de Estado da Saúde de São Paulo. Levantamento epidemiológico em saúde bucal Estado de São Paulo, 1998. São Paulo: Faculdade de Saúde Pública, Universidade de São Paulo; 1999.

32. Secretaria de Estado da Saúde de São Paulo/Faculdade de Saúde Pública, Universidade de São Paulo. Condições de saúde bucal no Estado de São Paulo em 2002. São Paulo: Secretaria de Estado da Saúde de São Paulo; 2002.

Recebido em 22/Jun/2005

Versão final reapresentada em 24/Out/2005 Aprovado em 08/Nov/2005 